\title{
Adverse outcomes from initiation of systemic corticosteroids for asthma: long-term observational study
}

This article was published in the following Dove Press journal: Journal of Asthma and Allergy

\author{
David B Price ${ }^{1,2}$ \\ Frank Trudo 3 \\ Jaco Voorham' \\ Xiao $\mathrm{Xu}^{4}$ \\ Marjan Kerkhof \\ Joanna Ling Zhi Jie' \\ Trung N $\operatorname{Tran}^{5}$ \\ 'Observational and Pragmatic \\ Research Institute, Singapore, \\ Singapore; ${ }^{2}$ Academic Primary Care, \\ Division of Applied Health Sciences, \\ University of Aberdeen, Aberdeen, \\ UK; ${ }^{3}$ Medical Affairs, AstraZeneca, \\ Wilmington, DE, USA; ${ }^{4}$ Global Payer \\ Evidence and Pricing, AstraZeneca, \\ Gaithersburg, MD, USA; ${ }^{5}$ Medical \\ Evidence and Observational Research, \\ AstraZeneca, Gaithersburg, MD, USA
}

Correspondence: David B Price Academic Primary Care, Division of Applied Health Sciences, University of Aberdeen, Polwarth Building, Foresterhill, Aberdeen AB25 2ZD, UK

Tel +65 68029724

Email dprice@opri.sg
Purpose: Prior work suggests a threshold of four courses/year of systemic corticosteroid (SCS) therapy is associated with adverse consequences. The objective of this study was to investigate the onset of adverse outcomes beginning at SCS initiation in a broad asthma population.

Patients and methods: This historical matched cohort study utilized anonymized, longitudinal medical record data (1984-2017) of patients ( $\geq 18$ years) with active asthma. Matched patients with first SCS prescription (SCS arm) and no SCS exposure (non-SCS arm) were followed until first outcome event. Associations between time-varying exposure measures and onset of 17 SCS-associated adverse outcomes were estimated using Cox proportional hazard regression, adjusting for confounders, in separate models.

Results: We matched 24,117 pairs of patients with median record availability before SCS initiation of 9.9 and 8.7 years and median follow-up 7.4 and 6.4 years in SCS and non-SCS arms, respectively. Compared with patients in the non-SCS arm, patients prescribed SCS had significantly increased risk of osteoporosis/osteoporotic fracture (adjusted hazard ratio 3.11; 95\% CI 1.87-5.19), pneumonia $(2.68 ; 2.30-3.11)$, cardio-/cerebrovascular diseases $(1.53 ; 1.36-1.72)$, cataract (1.50; 1.31-1.73), sleep apnea $(1.40 ; 1.04-1.86)$, renal impairment $(1.36 ; 1.26-1.47)$, depression/ anxiety $(1.31 ; 1.21-1.41)$, type 2 diabetes $(1.26 ; 1.15-1.37)$, and weight gain $(1.14 ; 1.10-1.18)$. A dose-response relationship for cumulative SCS exposure with most adverse outcomes began at cumulative exposures of $1.0-2.5 \mathrm{~g}$ and for some outcomes at cumulative exposures of only $0.5-<\mathrm{g}$ (vs $>0-<0.5 \mathrm{~g}$ reference), equivalent to four lifetime SCS courses.

Conclusion: Our findings suggest urgent need for reappraisal of when patients need specialist care and consideration of nonsteroid therapy.

Keywords: adverse outcomes, asthma, cumulative exposure, oral corticosteroids, systemic corticosteroids

\section{Introduction}

Systemic corticosteroids (SCS) are commonly prescribed to treat patients with respiratory conditions such as asthma and to treat or reduce the risk of flare-ups of inflammatory conditions, such as rheumatologic and autoimmune diseases, allergic reactions, and inflammatory bowel disease. ${ }^{1,2}$ However, the concern with maintenance SCS use is increased risk of infections and cardiovascular events; of chronic conditions such as type 2 diabetes mellitus, osteoporosis, and cataracts; of metabolic effects such as weight gain; and of neuropsychiatric effects such as insomnia, depression, and behavioral disturbances. ${ }^{3-5}$ A dose-response relationship with SCS exposure has been documented for many of these events. ${ }^{6-9}$ Even short-term use of oral corticosteroids (OCS) in a large population-based study has been associated with increased rates of 
sepsis, thromboembolism, and fracture within 30 days of OCS initiation. ${ }^{10}$ Of note, decreases in serum cortisol and markers of bone formation, and changes in white blood cell counts, are evident within hours of oral prednisone administration to healthy subjects. ${ }^{11}$

SCS, usually prescribed orally, are a mainstay of treatment for asthma exacerbations and are often prescribed as part of daily maintenance therapy for patients with severe asthma. ${ }^{12}$ An increased risk of adverse outcomes associated with regular SCS exposure has been recorded in severe asthma populations. ${ }^{8,9}$ In a recent cross-sectional, on-treatment study in the UK, ${ }^{13} 93 \%$ of patients with severe asthma had one or more conditions linked to SCS exposure, including type 2 diabetes, osteoporosis, dyspeptic disorders (gastric/duodenal ulcers), and cataracts. There are little data available, however, regarding the onset of SCS-associated adverse outcomes from the time of SCS initiation in a broad asthma population not restricted to those with severe disease. Moreover, additional data are needed to help confirm the relationship between cumulative SCS exposure and these outcomes, and the threshold exposure above which the risk of comorbid disease onset meaningfully increases. ${ }^{5,14,15}$ Of note, $1 \mathrm{~g}$ of SCS is equivalent to four short courses of OCS at the usual doses for treating an asthma exacerbation. ${ }^{12}$ Existing work suggests that a threshold of four SCS courses per year is associated with adverse consequences in patients with asthma. ${ }^{13,15}$

The aim of this study was to investigate the impact of initiating SCS, and of SCS exposure, on the onset of known SCS-associated adverse outcomes over a minimum of 2 years in a broad population of patients with active asthma of all levels of severity. Patients included in these analyses met the a priori definition for active asthma, which included a diagnostic Read code recorded within 1 year before or after (and including) the index date and at least two prescriptions for asthma medication in this period (reliever and/or controller medication). The prespecified adverse outcomes of interest included type 2 diabetes mellitus, cardio-/cerebrovascular diseases (myocardial infarction, heart failure, and stroke), dyslipidemia, hypertension, weight gain, osteoporosis, osteoporotic fracture, glaucoma, cataract, renal impairment, peptic ulcer disease, depression/anxiety, sleep apnea, and pneumonia.

\section{Patients and methods}

\section{Study design overview and data sources}

This was a historical matched cohort study of patients with active asthma comparing those initiating SCS (SCS arm) with those not exposed to SCS (non-SCS arm). The study comprised a minimum 1-year baseline period and a minimum 2-year follow-up period, on either side of an index date (Figure 1). The index date for patients in the SCS arm was the date of their first recorded prescription for parenteral or OCS, whereas the index date for those in the non-SCS arm was the nearest general practitioner (GP) visit to the matched case index date.

We utilized anonymized, longitudinal medical record data (January 1984 through January 2017) drawn from the Optimum Patient Care Research Database (OPCRD) ${ }^{16}$ and the Clinical Practice Research Datalink (CPRD). ${ }^{17}$ These large databases both include routinely collected data from medical records centralized in the UK at each patient's GP, such as diagnoses, prescriptions, clinical investigations, and hospital referrals and admissions. The OPCRD included over 3 million active patient records at the time of the study and the CPRD, over 5 million active patient records.

The study adhered to standards recommended for observational research (details in Supplementary material). ${ }^{18}$ The protocol was approved by the CPRD Independent Scientific Advisory Committee (ISAC reference number 17_002) and the Anonymized Data Ethics Protocols and Transparency committee (ADEPT Approval Reference ADEPT0117), the independent scientific advisory committee for the OPCRD. ${ }^{16}$ No patient-identifying information was accessible during the study.

\section{Patient population}

Patients eligible for the study were $\geq 18$ years of age and had active asthma and $\geq 3$ years of continuous practice records (including $\geq 1$-year baseline and $\geq 2$-year follow-up periods). Active asthma was defined as a diagnostic Read code recorded within 1 year before or after (and including) the index date and at least two prescriptions for asthma medication in this period (reliever and/or controller medication). Patients in the SCS arm had to have asthma as the most likely indication for their first SCS prescription plus $\geq 1$ additional prescription(s) for SCS within the next 18 months. A first SCS prescription was defined as no recorded prescription ever before the index date in the available data.

Patients were excluded from the analyses if their records contained a tamoxifen prescription for breast cancer at any time or diagnostic codes for adrenal insufficiency at any time or for cancer within 5 years before or 3 months after the index date. In addition, for the non-SCS arm, patients were excluded if they ever had any recorded prescriptions of SCS in the available data. Exclusion criteria for specific outcome (risk) cohorts are listed in Table S1. 


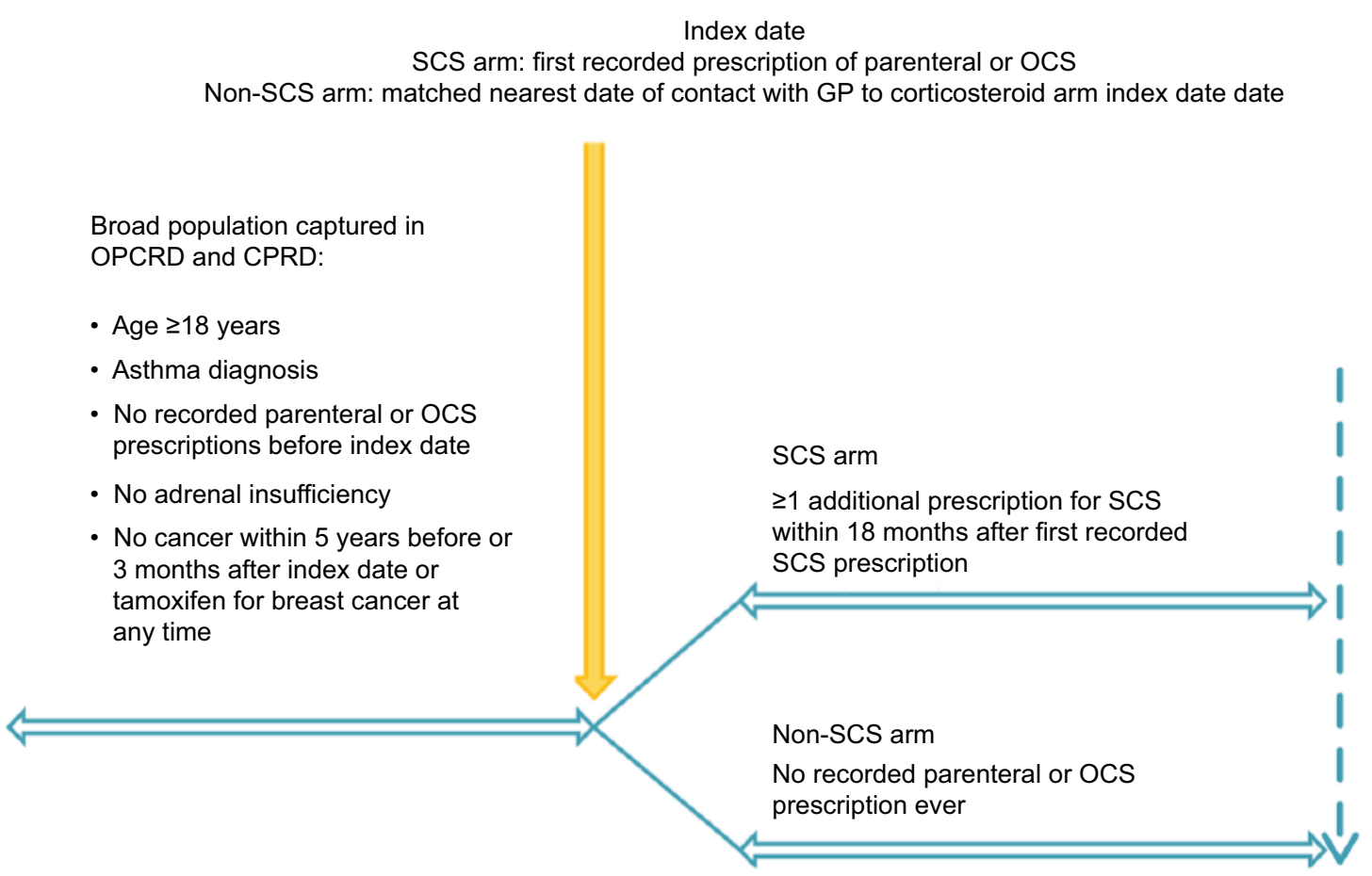

For time-to-onset analysis: Patients followed until the first occurrence of any of the following: onset of outcome condition or death, leaving the general practice, end of available records, or last date of extraction

$\begin{array}{lc}\text { Baseline period } & \text { Follow-up period } \\ \text { (minimum 1 year) } & \text { (minimum 2 years) }\end{array}$

Figure I Study design.

Abbreviations: CPRD, Clinical Practice Research Datalink; GP, general practitioner; OCS, oral corticosteroid; OPCRD, Optimum Patient Care Research Database; SCS, systemic corticosteroid.

\section{Study variables and outcomes}

The exposure of interest was SCS identified as any prescription for systemic prednisolone, methylprednisolone, prednisone, betamethasone, dexamethasone, hydrocortisone, or cortisone acetate, either as monotherapy or combination therapy. For patients in the SCS arm, we estimated the cumulative exposure of SCS (in grams, prednisolone-equivalent) and mean daily exposure ( $\mathrm{mg} /$ day) calculated as cumulative prednisolone-equivalent exposure divided by follow-up duration (Supplementary material).

The adverse outcomes (Table 1) were analyzed for patients who had no clinical diagnosis or other indications (medication use, laboratory measurements) specific to each given outcome before the index date (Table S1). Thus, separate risk cohorts were constructed for each adverse outcome under study.

\section{Patient matching}

Matching within the CPRD was conducted first, followed by matching in the OPCRD. Patients from the OPCRD who had identical values on the following variables as patients from the CPRD were removed from the combined database: index date, age, sex, practice joining date, practice leaving date, and asthma diagnosis; while duplicate controls were identified using index date, age, sex, practice joining date, and practice leaving date.

We first conducted 1:1 matching for sex, diagnosis of asthma, the index date (maximum distance of 182 days), and the availability of Hospital Episode Statistics (HES) linkage. The HES data, linked to CPRD, were used to identify hospital-related events. ${ }^{19} \mathrm{We}$ then retained only the patients with active asthma (see above for definition), together with their matched counterparts.

\section{Statistical analysis}

Descriptive statistics of all baseline characteristics were computed in the matched sample. We evaluated the quality of the matching using the standardized mean difference ${ }^{20}$ in combination with the bias potential ${ }^{21}$ (details in Supplementary material).

The incidence rate of each adverse outcome in the SCS and non-SCS arms, as cases per 100 patient-years of 
Table I Outcomes evaluated after the index date in each risk cohort

\begin{tabular}{|c|c|}
\hline Outcome & Definition applied after the index date (during follow-up period) ${ }^{a}$ \\
\hline Type 2 diabetes mellitus & $\begin{array}{l}\text { - Diagnosis of type } 2 \text { diabetes mellitus and/or } \\
\text { - One or more prescriptions for antidiabetic medication }{ }^{\mathrm{b}} \text { and/or } \\
\text { - HbAlc value } \geq 6.5 \%\end{array}$ \\
\hline Cardio-/cerebrovascular disease & - Myocardial infarction, heart failure, or stroke diagnosis \\
\hline Myocardial infarction & - Myocardial infarction diagnosis \\
\hline Heart failure & - Heart failure diagnosis \\
\hline Cerebrovascular accident & - Cerebrovascular accident diagnosis \\
\hline Dyslipidemia & $\begin{array}{l}\text { - Dyslipidemia diagnosis and/or total cholesterol reading }>6.5 \mathrm{mmol} / \mathrm{L} \text {, and/or low-density lipoprotein reading } \\
>4 \mathrm{mmol} / \mathrm{L} \text {, and/or triglyceride reading } \geq 2.3 \mathrm{mmol} / \mathrm{L}\end{array}$ \\
\hline Hypertension & - Hypertension diagnosis \\
\hline Weight gain & - An increase in $\mathrm{BMI}$ of $\geq \mathrm{I} \mathrm{kg} / \mathrm{m}^{2}$ compared with the last $\mathrm{BMI}$ in the 5 years before the index date \\
\hline Osteoporosis diagnosis & - Osteoporosis diagnosis \\
\hline $\begin{array}{l}\text { Osteoporosis and osteoporotic } \\
\text { fracture }\end{array}$ & $\begin{array}{l}\text { - Osteoporosis diagnosis AND } \\
\text { - Likely osteoporotic fracture (hip, wrist, or vertebral fracture) }\end{array}$ \\
\hline Glaucoma & - Glaucoma diagnosis and/or treatment for glaucoma \\
\hline Cataract & - Cataract diagnosis or surgery for cataract \\
\hline Renal impairment & - Chronic kidney disease stage $3 a+$ diagnosis, or eGFR $<60 \mathrm{~mL} / \mathrm{min} / 1.72 \mathrm{~m}^{2}$, or dialysis or renal transplant \\
\hline Peptic ulcer & - Peptic ulcer diagnosis \\
\hline Depression/anxiety & - Depression/anxiety diagnosis or depression/anxiety diagnosis and prescription of antidepressants \\
\hline Sleep apnea & - Sleep apnea diagnosis and/or referral to sleep clinic and use of a continuous positive airway pressure device \\
\hline Pneumonia & - Pneumonia diagnosis \\
\hline
\end{tabular}

Notes: ${ }^{a}$ Diagnoses were defined as diagnostic Read codes recorded after the index date. See Table SI for exclusion criteria specific to each risk cohort. ${ }^{\mathrm{b}} \mathrm{Patients}$ with a diagnosis of polycystic ovary syndrome (PCOS) and $\geq I$ metformin prescriptions in the follow-up period were excluded because metformin is given as treatment for PCOS. Abbreviations: BMI, body mass index; eGFR, estimated glomerular filtration rate.

follow-up (100 pt-yr), was compared using the incidence rate difference and the incidence rate ratio (IRR) with $95 \%$ CIs. Each patient was followed until the first occurrence of the outcome of interest and was censored at death or the end of available records. We used conditional multivariable Cox proportional hazard models with $95 \%$ CIs to compare the risk of adverse outcomes between SCS and non-SCS arms and account for the clustered nature of the matched data.

In the SCS arm, the cumulative SCS exposure and the mean daily exposure, both as continuous and categorized variables, were modeled as time-dependent exposures to assess their association with the development of each outcome using a time-to-event design. We used multivariable Cox proportional hazard models with $95 \%$ CIs to determine risk of each outcome from SCS exposure.

For each outcome model, three sets of confounding variables were defined a priori based on clinical knowledge for each risk cohort (Table S2): (1) variables that were always adjusted for, (2) variables available for bias potential assessment during model building (process described in Supplementary material), and (3) variables that were not considered in the models. Confounders applied to the adjusted models are provided in Table S3.
Dataset creation was performed using SAS version 9.4 (SAS Institute, Cary, NC, USA), and statistical analyses were conducted using Stata SE version 14.2 and Stata MP/6 version 15.0 (StataCorp, College Station, TX, USA). Results were considered statistically significant if $P \leq 0.05$.

\section{Results \\ Patients}

Overall, the databases contained 307,213 matched pairs of patients. Of these, 117,409 (38\%) patients in the SCS arm were prescribed SCS for asthma, 58,577 (19\%) for COPD, and $1,536(0.5 \%)$ for asthma and COPD. We analyzed data for the 24,117 matched pairs (48,234 total patients) who had study-defined active asthma. This represented $21 \%$ of the 117,409 matched pairs of patients with an SCS prescription for asthma. More than $99 \%$ of patient pairs were matched on the same index date (ie, the date of first SCS prescription for patients in the SCS arm). For 18,291 (76\%) patient pairs, the index date was in the year 2000 or later, and for $13,522(56 \%), 2005$ or later.

In the SCS arm, the mean record availability before initiation of SCS (preindex date) was 14.3 years (median 9.9 years; IQR 4.1-20.0) and in the non-SCS arm, the mean 
preindex record availability was 12.8 years (median 8.7 years; IQR 3.7-18.2). Overall, 17,246 (72\%) and 17,540 (73\%) patient pairs in SCS and non-SCS arms, respectively, had 4 or more years of medical record data available before the index date. The mean follow-up time (postindex date) was 8.7 years in the SCS arm (median 7.4 years; IQR 4.5-11.2) and 7.7 years in the non-SCS arm (median 6.4 years; IQR 3.8-10.0).

The mean ages of the matched SCS and non-SCS arms were 49 (SD 17) and 44 (SD 18) years, respectively; and $15,585(65 \%)$ in each cohort were women (Table 2). In the SCS arm, more patients were obese $(5,570$ [33\%] vs 4,524 [26\%] in non-SCS arm) and current smokers $(8,604$ [37\%] vs 7,373 [32\%]) at baseline. A greater percentage of patients in the SCS arm were treated with antibiotics for one or more infections, respectively, during the baseline year (Table 2).

Of 305,110 SCS prescriptions included in the analyses, the majority $(299,785 ; 98 \%)$ were for OCS and 5,325 (2\%) were for parenteral corticosteroids.

\section{Incidence of adverse outcomes by SCS use}

In all risk cohorts, patients in the SCS arm, irrespective of treatment regimen, experienced a significantly higher incidence of the study outcome compared with the non-SCS arm (Table 3). Pneumonia had the greatest relative incidence with SCS use (IRR 2.92; 95\% CI 2.60-3.29). Osteoporosis diagnosis and fracture had the second greatest relative incidence with SCS use (IRR 2.84; 95\% CI 2.05-3.98), even though it was the least common outcome, with 0.085 and 0.030 events per 100 person-years recorded in SCS and non-SCS arms, respectively. The onset of type 2 diabetes was one of the most common adverse outcomes, with 1.047 vs 0.615 events per 100 person-years for patients with and without SCS use, respectively, and an IRR of 1.70 (1.58-1.84) for SCS vs non-SCS arms (Table 3).

For the SCS arm (vs non-SCS arm), the adjusted risk of the onset of an adverse outcome was significantly greater for 13 of 17 outcomes, namely, excepting the onset of dyslipidemia, hypertension, glaucoma, and peptic ulcer. For these 13 outcomes, the adjusted risk in the SCS arm compared with the non-SCS arm ranged from 1.14 times greater for weight gain (BMI increase of $\geq 1 \mathrm{~kg} / \mathrm{m}^{2}$ ) to 3.11 times greater for new osteoporosis diagnosis/osteoporotic fracture (Figure 2).

\section{Cumulative SCS exposure and adverse outcomes in the SCS arm}

A positive dose-response relationship was present between categorized, cumulative SCS exposure and adverse outcome in most risk cohorts, except dyslipidemia, hypertension, weight increase, and glaucoma (Figure 3). This positive dose-response relationship was evident at the lowest exposure categories, with increasing risk of most outcomes evident and becoming statistically significant when progressing from a cumulative SCS exposure of 0.5 to $<1 \mathrm{~g}$ to a cumulative SCS exposure of 1.0 to $<2.5 \mathrm{~g}$ compared with the reference category of $>0$ to $<0.5 \mathrm{~g} \mathrm{SCS}$ (Figure 3). For example, the risk of type 2 diabetes onset was $16 \%$ higher at cumulative SCS exposure of 0.5 to $<1 \mathrm{~g}$ (vs reference $>0-<0.5 \mathrm{~g}$ : adjusted hazard ratio [aHR] 1.16; 95\% CI 1.01-1.34) and increased to $37 \%$ at cumulative SCS exposure of 1.0 to $<2.5 \mathrm{~g}$ (aHR 1.37; 95\% CI 1.18-1.58). Further details for each SCS exposure category and risk cohort are presented in Table S4.

For cumulative SCS dose as a continuous variable, a significantly increased risk was recorded for all outcomes except hypertension and weight gain per 1-g increase, with most increases in the range of $1.02-1.03$ times $(2 \%-3 \%$; Table S4).

\section{Mean daily SCS exposure and adverse outcomes in the SCS arm}

A positive dose-response relationship between mean daily SCS exposure and each outcome studied was also evident compared with the reference category of $>0$ to $<0.5 \mathrm{mg} /$ day exposure, as summarized in Table S5. At a mean daily SCS exposure of 0.5 to $<2.5 \mathrm{mg} /$ day, the aHR ranged from 1.77 (95\% CI 1.59-1.96) for hypertension to 3.10 (95\% CI 2.64-3.63) for osteoporosis diagnosis; at a mean daily SCS exposure of $\geq 7.5 \mathrm{mg} /$ day, the aHR ranged up to $14.7(95 \%$ CI 11.12-19.49) for osteoporosis diagnosis. For every 5-mg increase in mean daily exposure, the adjusted risk of each outcome increased from $21 \%$ to $70 \%$ (Table S5).

\section{Discussion}

In this large historical cohort study, we found that patients with active asthma experienced a significantly higher onset of prespecified adverse outcomes after their first SCS prescription than matched controls not exposed to SCS. Patients in the SCS arm were at significantly increased risk of developing osteoporosis/osteoporotic fracture, pneumonia, cardio-/ cerebrovascular diseases (including myocardial infarction, heart failure, cerebrovascular accident), cataract, sleep apnea, renal impairment, depression/anxiety, type 2 diabetes, and weight gain. Moreover, among patients in the SCS arm, we found a positive dose-response relationship with multiple adverse outcomes regarding both cumulative SCS exposure and mean daily SCS exposure. This relationship was both 
Table 2 Baseline characteristics of the matched treatment arms

\begin{tabular}{|c|c|c|c|}
\hline & $\begin{array}{l}\text { Non-SCS } \\
(n=24,117)\end{array}$ & $\begin{array}{l}\text { SCS } \\
(n=24,117)\end{array}$ & SMD (\%) \\
\hline Sex, female ${ }^{a}$ & $15,585(65 \%)$ & $15,585(65 \%)$ & 0.0 \\
\hline Age at index date (years) & $43.9(18.1)$ & $48.7(16.9)$ & 27.4 \\
\hline $18-40$ & I I,958 (50\%) & $8,519(35 \%)$ & 23.5 \\
\hline $4 I-60$ & $7,072(29 \%)$ & $9,055(38 \%)$ & \\
\hline $61-80$ & $4,342(18 \%)$ & $5,893(24 \%)$ & \\
\hline$>80$ & $745(3 \%)$ & $650(3 \%)$ & \\
\hline First SCS and matched non-SCS, median year (IQR) ${ }^{\mathrm{a}}$ & $2,005(9.0)$ & $2,005(9.0)$ & 0.0 \\
\hline Available HES data linkage $^{a}$ & $9,413(39 \%)$ & $9,413(39 \%)$ & 0.0 \\
\hline Body mass index $\left(\mathrm{kg} / \mathrm{m}^{2}\right), \mathrm{n}(\% \text { nonmissing })^{\mathrm{b}}$ & $17,466(72 \%)$ & $16,810(70 \%)$ & 18.8 \\
\hline$<18.5$ & $435(2 \%)$ & $328(2 \%)$ & \\
\hline$\geq 18.5$ to $<25$ & $6,996(40 \%)$ & $5,439(32 \%)$ & \\
\hline$\geq 25$ to $<30$ & $5,51 I(32 \%)$ & $5,473(33 \%)$ & \\
\hline$\geq 30$ & $4,524(26 \%)$ & $5,570(33 \%)$ & \\
\hline Smoking status, $\mathrm{n}$ (\% nonmissing) ${ }^{\mathrm{b}}$ & 23,281 (97\%) & $23,587(98 \%)$ & 13.7 \\
\hline Current smoker & $7,373(32 \%)$ & $8,604(36 \%)$ & \\
\hline Ex-smoker & $4,06 \mid(17 \%)$ & $4,773(20 \%)$ & \\
\hline Nonsmoker & II,847 (5 I\%) & $10,210(43 \%)$ & \\
\hline \multicolumn{4}{|l|}{ GINA step ${ }^{c, d}$} \\
\hline No medication & $2,302(10 \%)$ & $4,454(18 \%)$ & 2.4 \\
\hline Step I & $4,590(19 \%)$ & $3,970(16 \%)$ & \\
\hline Step 2 & $9,512(39 \%)$ & $6,386(26 \%)$ & \\
\hline Step 3 & $4,761(20 \%)$ & $4,972(21 \%)$ & \\
\hline Step 4 & $2,952(12 \%)$ & $4,335(18 \%)$ & \\
\hline High-dose ICS (mean dose $>500 \mu \mathrm{g} /$ day) ${ }^{\mathrm{b}}$ & $1,029(4 \%)$ & $1,676(7 \%)$ & 11.7 \\
\hline \multicolumn{4}{|l|}{ FDC ICS/LABA: ICS daily dose $(\mu g / \text { day })^{c}$} \\
\hline 0 & $7,109(30 \%)$ & $8,692(36 \%)$ & 2.4 \\
\hline$>0-400$ & $15,284(63 \%)$ & $12,792(53 \%)$ & \\
\hline$>400-800$ & $\mathrm{I}, 357(6 \%)$ & $2,002(8 \%)$ & \\
\hline$>800$ & $367(2 \%)$ & $631(3 \%)$ & \\
\hline \multicolumn{4}{|l|}{ SABA $(\mu g / \text { day })^{c}$} \\
\hline 0 & $5,062(21 \%)$ & $4,76 \mathrm{I}(20 \%)$ & 7.3 \\
\hline $1-100$ & $4,247(18 \%)$ & $4,614(19 \%)$ & \\
\hline $10 I-200$ & $6,214(26 \%)$ & $5,327(22 \%)$ & \\
\hline $20 I-400$ & $4,558(19 \%)$ & $4,370(18 \%)$ & \\
\hline$>400$ & $4,036(17 \%)$ & $5,045(21 \%)$ & \\
\hline \multicolumn{4}{|l|}{ Infections treated with antibiotics ${ }^{c}$} \\
\hline 0 & $20,476(85 \%)$ & $14,799(61 \%)$ & 49.0 \\
\hline 1 & $2,343(10 \%)$ & $5,747(24 \%)$ & \\
\hline 2 & $868(4 \%)$ & $2,361(10 \%)$ & \\
\hline$\geq 3$ & $430(2 \%)$ & $\mathrm{I}, 210(5 \%)$ & \\
\hline \multicolumn{4}{|l|}{ Conditions recorded ever, before index date } \\
\hline Type 2 diabetes mellitus ${ }^{e}$ & $\mathrm{I}, 223(5 \%)$ & $931(4 \%)$ & 5.9 \\
\hline Cardio-/cerebrovascular disease & $\mathrm{I}, 037(4 \%)$ & $1,016(4 \%)$ & 0.4 \\
\hline Myocardial infarction & $420(2 \%)$ & $458(2 \%)$ & 1.2 \\
\hline Heart failure & $294(1 \%)$ & $255(1 \%)$ & 1.5 \\
\hline Cerebrovascular accident & $47 \mathrm{I}(2 \%)$ & $426(2 \%)$ & 1.4 \\
\hline Dyslipidemia & $2,964(12 \%)$ & $3,211(13 \%)$ & 3.1 \\
\hline Hypertension & $3,754(16 \%)$ & $4,145(17 \%)$ & 4.4 \\
\hline Osteoporosis & $319(1 \%)$ & $383(2 \%)$ & 2.2 \\
\hline Osteoporosis or osteoporotic fracture & $\mathrm{I}, 400(6 \%)$ & $1,565(6 \%)$ & 2.8 \\
\hline Glaucoma & $258(1 \%)$ & $293(1 \%)$ & 1.4 \\
\hline Cataract diagnosis or surgery & $727(3 \%)$ & $768(3 \%)$ & 1.0 \\
\hline Renal impairment ${ }^{f}$ & $2,004(8 \%)$ & $2,204(9 \%)$ & 2.0 \\
\hline Peptic ulcer & $427(2 \%)$ & $553(2 \%)$ & 3.7 \\
\hline
\end{tabular}


Table 2 (Continued)

\begin{tabular}{|c|c|c|c|}
\hline & $\begin{array}{l}\text { Non-SCS } \\
(n=24, I \mid 7)\end{array}$ & $\begin{array}{l}\text { SCS } \\
(n=24,|| 7)\end{array}$ & SMD (\%) \\
\hline Depression/anxiety & $6,109(25 \%)$ & $7,088(29 \%)$ & 9.1 \\
\hline Sleep apnea & $69(0.3 \%)$ & $71(0.3 \%)$ & 0.2 \\
\hline Pneumonia in the prior year ${ }^{c}$ & $68(0.3 \%)$ & I I 7 (0.5\%) & 3.3 \\
\hline \multicolumn{4}{|l|}{ Charlson comorbidity index ${ }^{g}$} \\
\hline $0-4$ & $10,778(45 \%)$ & I I,070 (46\%) & 5.6 \\
\hline $5-8$ & $10,325(43 \%)$ & $9,995(41 \%)$ & \\
\hline $9-12$ & $827(3 \%)$ & $804(3 \%)$ & \\
\hline $13-16$ & $829(3 \%)$ & $952(4 \%)$ & \\
\hline$\geq 17$ & $1,358(6 \%)$ & $1,296(5 \%)$ & \\
\hline
\end{tabular}

Notes: Unless otherwise noted, data are $\mathrm{n}(\%)$, mean (SD), or median (IQR). Percentages may not add up to I00 because of rounding. ${ }^{\mathrm{a}}$ Matching variable. ${ }^{\mathrm{b}}$ The closest body mass index within 5 years before and the smoking status closest to index date were included. High-dose ICS (fluticasone propionate-equivalent dose) refers to the last

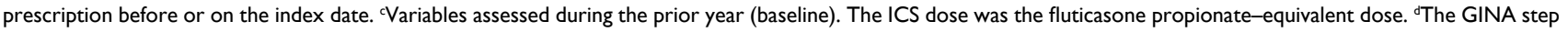
was based on the last asthma medication(s) prescribed during the baseline year, including the index date. For the determination of GINA step in the SCS arm, "no medication" was defined as no asthma-related medication other than SCS prescribed at the index date. No patients were receiving biologics or at GINA step 5 . eType 2 diabetes mellitus diagnostic Read code or $\mathrm{HbAlc} \geq 6.5 \%$ recorded twice or more. 'Stage $3 \mathrm{a}$ or higher at most recent assessment before the index date. ${ }^{8} \mathrm{Greater}$ scores predict increased 10 year risk of mortality for people with multiple comorbidities.

Abbreviations: FDC, fixed-dose combination; GINA, Global Initiative for Asthma; HES, Hospital Episode Statistics; ICS, inhaled corticosteroid; LABA, long-acting $\beta$-agonist; SABA, short-acting $\beta$-agonist; SMD, standardized mean difference; SCS, systemic corticosteroid.

Table 3 Incidence of outcomes of interest in the matched non-SCS and SCS arms

\begin{tabular}{|c|c|c|c|c|c|c|c|c|c|c|}
\hline \multirow[t]{2}{*}{ Risk cohort } & \multicolumn{4}{|c|}{ Non-SCS } & \multicolumn{4}{|l|}{ SCS } & \multirow{2}{*}{$\begin{array}{l}\text { IR difference } \\
(95 \% \mathrm{Cl})\end{array}$} & \multirow{2}{*}{$\begin{array}{l}\text { Unadjusted IR } \\
\text { ratio }(95 \% \mathrm{Cl})\end{array}$} \\
\hline & $\overline{\mathbf{N}}$ & Pt-yr & $\begin{array}{l}\text { No. } \\
\text { events }\end{array}$ & $\begin{array}{l}\text { IR (per } \\
100 \text { pt-yr) }\end{array}$ & $\overline{\mathbf{N}}$ & Pt-yr & $\begin{array}{l}\text { No. } \\
\text { events }\end{array}$ & $\begin{array}{l}\text { IR (per } \\
\text { I00 pt-yr) }\end{array}$ & & \\
\hline Type 2 diabetes mellitus & 21,808 & 166,756 & 1,025 & 0.615 & 21,808 & 183,458 & 1,921 & 1.047 & $0.432(0.372-0.493)$ & $1.70(1.58-1.84)$ \\
\hline $\begin{array}{l}\text { Cardio-/cerebrovascular } \\
\text { disease }\end{array}$ & 22,112 & 169,323 & 826 & 0.488 & 22,112 & 185,195 & $\mathrm{I}, 774$ & 0.958 & $0.470(0.4 \mid 4-0.526)$ & $1.96(1.8 I-2.14)$ \\
\hline Myocardial infarction & 23,251 & 178,974 & 298 & 0.167 & 23,251 & 199,249 & 646 & 0.324 & $0.158(0.126-0.189)$ & $1.95(1.69-2.24)$ \\
\hline Heart failure & 23,570 & $|81,20|$ & 349 & 0.193 & 23,570 & 200,790 & 858 & 0.427 & $0.235(0.200-0.270)$ & $2.22(1.96-2.52)$ \\
\hline $\begin{array}{l}\text { Cerebrovascular } \\
\text { accident }\end{array}$ & 23,228 & 178,454 & 451 & 0.253 & 23,228 & 198,519 & 823 & 0.415 & $0.162(0.125-0.199)$ & $1.64(1.46-1.84)$ \\
\hline Dyslipidemia & 16,903 & 129,550 & 2,346 & 1.811 & 16,903 & 139,700 & 3,523 & 2.522 & $0.711(0.600-0.822)$ & $1.39(1.32-1.47)$ \\
\hline Hypertension & 12,248 & 95,871 & 986 & 1.028 & 12,248 & 104,340 & 1,626 & 1.558 & $0.530(0.431-0.629)$ & $1.52(1.40-1.64)$ \\
\hline $\begin{array}{l}\text { Weight gain (BMI increase } \\
\left.\geq 1 \mathrm{~kg} / \mathrm{m}^{2}\right)\end{array}$ & 12,894 & 53,496 & 6,718 & 12.558 & 12,894 & 52,680 & 7,677 & 14.573 & $2.015(1.572-2.458)$ & $1.16(1.12-1.20)$ \\
\hline Osteoporosis diagnosis & 23,422 & $|79,97|$ & 382 & 0.212 & 23,422 & 198,056 & 964 & 0.487 & $0.274(0.237-0.3 \mid 2)$ & $2.29(2.03-2.59)$ \\
\hline $\begin{array}{l}\text { Osteoporosis diagnosis } \\
\text { and fracture }\end{array}$ & 21,251 & 166,344 & 50 & 0.030 & $21,25 I$ & 186,270 & 159 & 0.085 & $0.055(0.040-0.07 I)$ & $2.84(2.05-3.98)$ \\
\hline Glaucoma & 23,424 & 180,109 & 273 & 0.152 & 23,424 & 201,118 & 433 & 0.215 & $0.064(0.037-0.091)$ & $1.42(1.22-1.66)$ \\
\hline Cataract & 22,648 & 173,139 & 830 & 0.479 & 22,648 & $|90,05|$ & I,736 & 0.913 & $0.434(0.380-0.488)$ & 1.91 (1.75-2.07) \\
\hline Renal impairment & 20,137 & 152,844 & $\mathrm{I}, 758$ & 1.150 & 20,137 & 162,698 & 3,215 & 1.976 & $0.826(0.739-0.913)$ & $1.72(1.62-1.82)$ \\
\hline Peptic ulcer & 23,149 & 177,768 & 135 & 0.076 & 23,149 & 199,008 & 219 & 0.110 & $0.034(0.015-0.054)$ & $1.45(1.16-1.81)$ \\
\hline Depression/anxiety & 7,961 & 62,801 & 1,257 & 2.002 & 7,961 & 66,924 & $\mathrm{I}, 554$ & 2.322 & $0.320(0.16 \mathrm{I}-0.480)$ & $1.16(1.08-1.25)$ \\
\hline Sleep apnea & 23,980 & 184,930 & 84 & 0.045 & 23,980 & 207,072 & 205 & 0.099 & $0.054(0.037-0.070)$ & $2.18(1.68-2.84)$ \\
\hline Pneumonia & 24,065 & 184,422 & 379 & 0.206 & 24,065 & 203,107 & 1,220 & 0.601 & $0.395(0.356-0.435)$ & $2.92(2.60-3.29)$ \\
\hline
\end{tabular}

Abbreviations: BMI, body mass index; IR, incidence rate; pt-yr, patient-years; SCS, systemic corticosteroid.

statistically significant and clinically important, as increased risks of adverse outcomes were seen at relatively low cumulative and mean daily SCS exposures.

We examined several categories of cumulative SCS exposure for patients in the SCS arm from the time of their first SCS prescription. Relative to the reference category of $>0$ to $<0.5 \mathrm{~g}$, a statistically significant increased risk for onset of type 2 diabetes (aHR 1.16) was evident at a cumulative exposure of only 0.5 to $<1 \mathrm{~g} \mathrm{SCS}$ and for multiple other adverse outcomes at cumulative exposure of 1.0 to $<2.5 \mathrm{~g} \mathrm{SCS}$. We note that $1 \mathrm{~g} \mathrm{SCS}$ is equivalent to four short courses of OCS at the usual doses for treating an asthma exacerbation. ${ }^{12}$ Thus, an important clinical, as well as practical, implication of our findings is that patients 


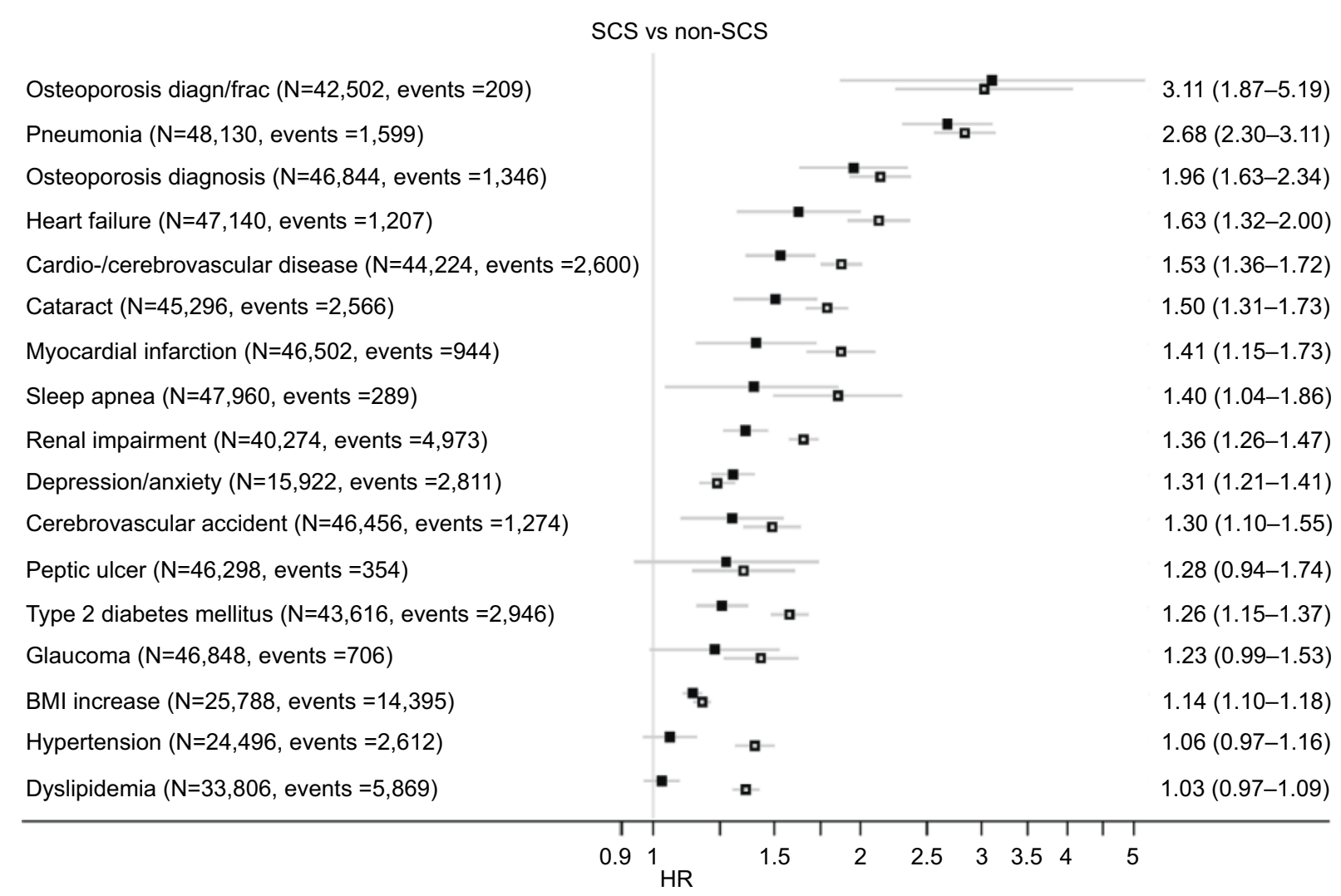

Figure 2 HR $(95 \% \mathrm{Cl})$ for each adverse outcome in the SCS arms (vs non-SCS arms). The open squares represent unadjusted, and the closed squares, adjusted results. The adjusted HRs ( $95 \% \mathrm{Cls}$ ) are shown on the right. See Table $\mathrm{S} 3$ for list of confounders.

Abbreviations: BMI, body mass index; SCS, systemic corticosteroid.

should be evaluated and considered for alternative treatment strategies early in the course of their asthma to avoid the need for OCS.

Although SCS are well-established drugs, available generically, and thus inexpensive, health-care resource utilization studies show a substantial impact from the associated adverse outcomes and their treatment. ${ }^{3,22,23}$ Furthermore, a positive dose-response relationship between long-term SCS exposure and health-care resource utilization and costs was recently found for patients with active asthma. ${ }^{23}$ The cost ratio $(95 \% \mathrm{CI})$ associated with SCS use compared with non-SCS use increased from $1.25(1.18-1.31)$ for $0.5-<2.5 \mathrm{mg} /$ day to $2.41(2.02-2.88)$ for $7.5-<15.0 \mathrm{mg} /$ day and $3.86(2.53-5.89)$ for $\geq 15.0 \mathrm{mg} /$ day. ${ }^{23}$ We found that most SCS was prescribed for respiratory conditions (for a total of 177,522 matched pairs of patients in the database, or 58\%), primarily for asthma. Results of the continuous exposure model for a 1-g increase in cumulative exposure to SCS might suggest that effects of exposure are low, with significant HRs in the 1.02-1.03 range; however, it is important to note that this model incorporates the assumption that average effect is independent of the initial exposure level, meaning that a $1-\mathrm{g}$ increase from 0 to $1 \mathrm{~g}$ total dose would result in the same risk increase as an increase from 4 to $5 \mathrm{~g}$. The results from the categorical model show that this assumption does not hold true, as the HR for patients with 10-g cumulative exposure was more than two times greater than that at the lowest category of exposure for most adverse outcomes, whereas a $22 \%$ to $34 \%$ increased risk would be estimated from the continuous model $\left(1.02^{10}-1.03^{10}\right)$.

The results from the daily exposure analyses show a clear, positive dose-response relationship consistent with that seen with cumulative exposure. For example, the risk of onset of type 2 diabetes is almost nine times greater for patients prescribed $>7.5 \mathrm{mg} /$ day vs $0-<0.5 \mathrm{mg}$ /day. These findings, which incorporate both duration and dosage, are challenging to translate into a specific clinical recommendation; however, of note, we observed the increase at even very low daily exposures and can conclude that minimizing SCS exposure should be an important goal of asthma therapy. 

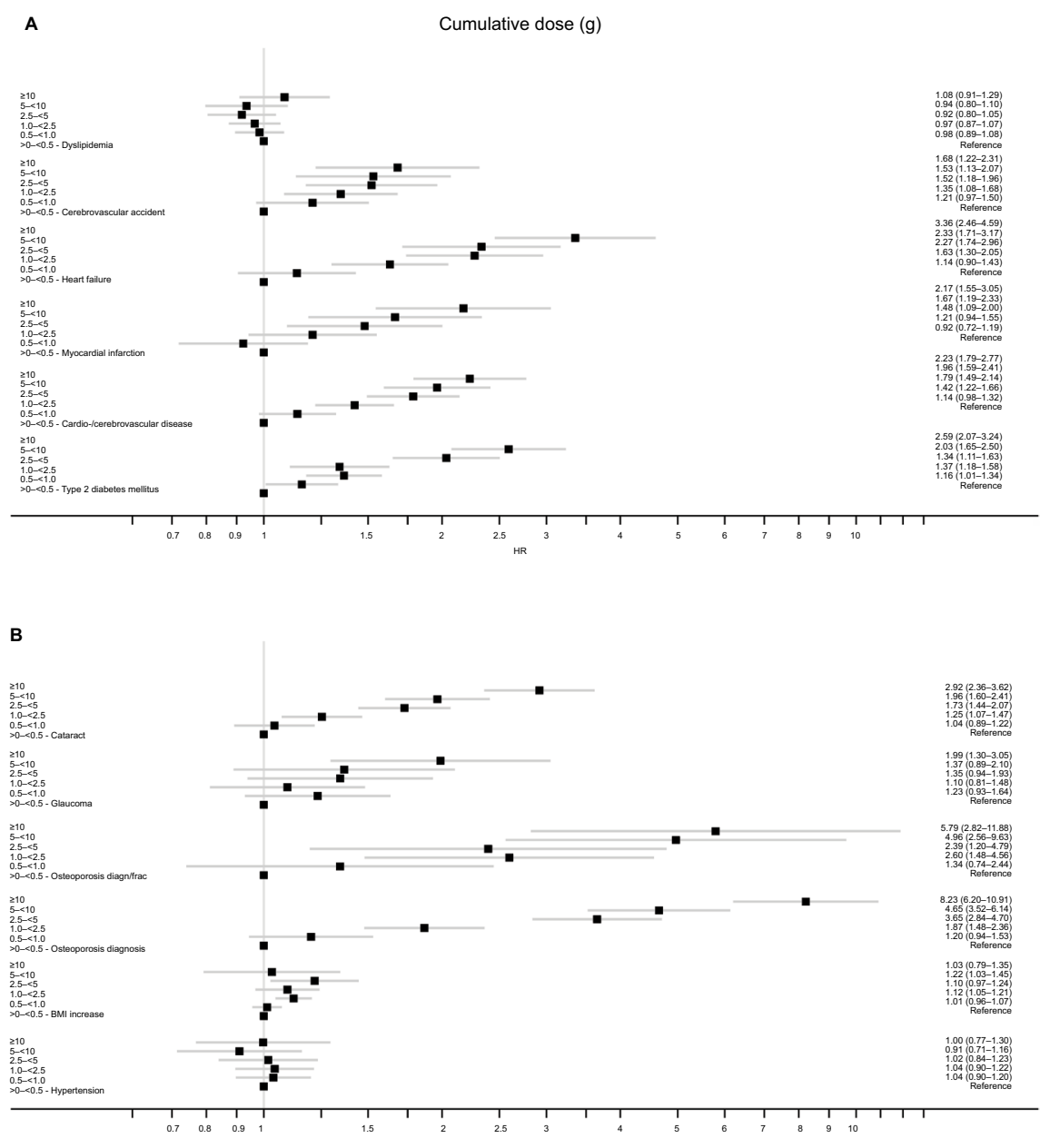

C

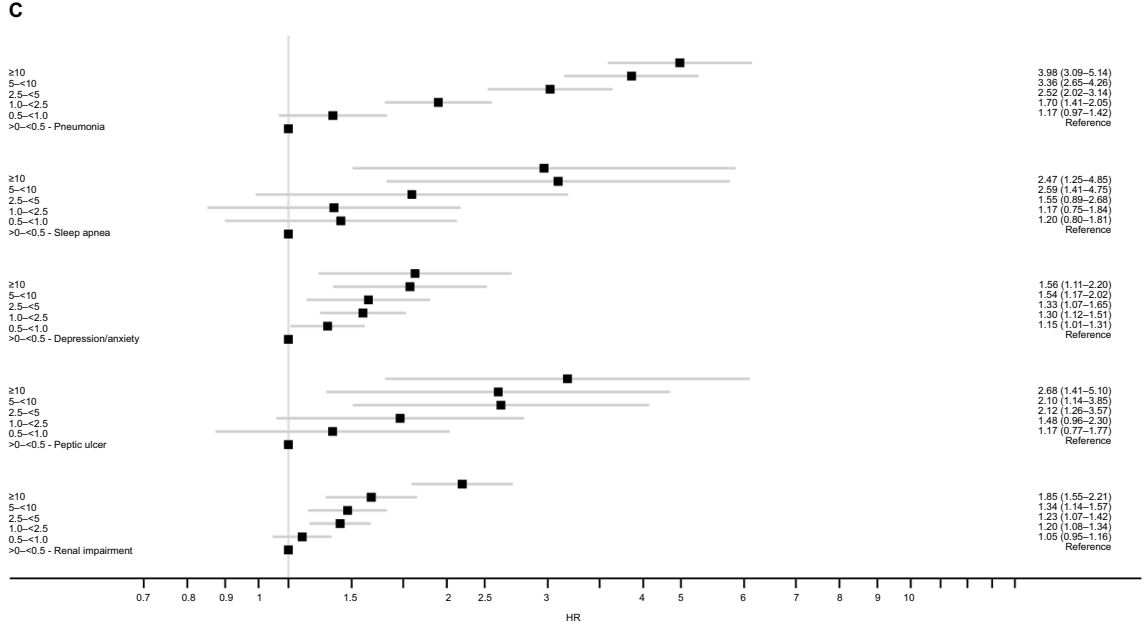

Figure 3 (A-C) Adjusted hazard ratio $(95 \% \mathrm{Cl})$ for each adverse outcome in the SCS arms for categorized, cumulative SCS exposures, compared with the reference category of $>0$ to $<0.5$-g cumulative exposure. See Table S3 for list of confounders. Abbreviations: BMI, body mass index; SCS, systemic corticosteroid.

Our findings support those of the recent study of Sullivan et $\mathrm{al},{ }^{15}$ while the high granularity data in the OPCRD and CPRD enabled us to further characterize the association of adverse outcomes with defined SCS exposure (doses) over time using the different exposure measures. We used an incident (new) user study design that allowed us to capture adverse outcomes of interest from the first SCS prescription. ${ }^{24}$ We followed patients from the time of SCS initiation until the 
time they experienced an event or were otherwise censored. The use of large UK databases containing longitudinal medical record data allowed us to examine a median of almost 10 years before the first SCS prescription to exclude prior SCS use, with subsequent follow-up of a median of 7 years, much longer than prior studies in asthma with reported mean and median follow-up periods of 1-5 years. ${ }^{7-9,15,25}$ Moreover, earlier studies examined effects of ongoing (rather than initiating) SCS exposure ${ }^{7,8,25-27}$ or ruled out previous exposure for only $\leq 12$ months before the index prescription. ${ }^{9,15}$

Most prior studies included limited patient populations, ie, those classified as having severe asthma ${ }^{7-9,25}$ or those prescribed SCS for prespecified lengths of time. ${ }^{26,27}$ Studies from the USA included low-income ${ }^{7,8}$ or employed ${ }^{15,25-28}$ populations, selective populations with limited or better-thanaverage access to care, respectively. Instead, the majority of people in the UK are registered with GPs, who administer primary care and serve as the gate-keeper for secondary care; thus, the CPRD and OPCRD include data from a broad, representative patient population, supporting the generalizability of our findings.

Our study has a number of other strengths. The OPCRD and CPRD are well-established databases whose large size, high data quality, and granularity enabled us to study the association between corticosteroid exposure and the risk of multiple different adverse outcomes. The data pertaining to drug use and disease diagnoses are recorded prospectively and therefore were not influenced by recall bias.

Study limitations include those applicable to all historical database studies, including the potential for errors in documentation, as the data collected were not intended for research purposes. This does, however, have the benefit of potentially eliminating researcher bias in the collection of the data. We matched patients on a small number of characteristics (sex, index date, and available HES linkage) and then adjusted for multiple confounders in the analyses. For the construction of the initial study cohort, more than 24 million non-SCS patients' records were available for matching. Adding age with a maximum caliper to the matching criteria would have resulted in a sizeable increase in computing time. Therefore, for practical reasons, it was decided to handle confounding by age at the analysis level. Age was always included as a confounder, and inhaled corticosteroid exposure was considered as a potential confounder, in each model. The patients in the SCS arm were on average 5 years older than those in the non-SCS arm, and a greater percentage of patients were obese and current smokers. The two arms differed in several other characteristics, suggesting a greater asthma-related disease burden for patients prescribed SCS, as would be expected. However, we do not consider asthma severity to be associated with the onset of adverse outcomes, with the possible exception of pneumonia; thus, we suspect these findings could not have introduced much bias. We handled residual confounding during model building, drawing on a large list of potential confounders assembled from extensive experience with observational research in respiratory disease. Nonetheless, there remains the possibility of residual confounding from unrecognized and/or unmeasured factors.

Information on SCS exposure was drawn from prescribing records, which could lead to some misclassification because we could not establish whether the prescriptions were dispensed (although prescribing records from the CPRD are considered reliable ${ }^{29}$ ) nor whether patients adhered to the SCS prescribed regimen. There is also the likelihood of bias in the detection of certain outcomes such as diabetes mellitus and osteoporosis in the SCS arm, as patients may be more likely to be screened for these conditions while receiving corticosteroid therapy. ${ }^{13}$ For about one-quarter of patients, there were fewer than 4 years of available history, which could have resulted in unaccounted prior SCS exposure and an underestimation of the true effects. Further potential for underestimation could have resulted from our inclusion of only the first outcome event, censoring patients after the onset of any outcome, thereby excluding recurrent events. Finally, the influence of obesity on outcomes could not be evaluated, as this was not a main study objective. Therefore, obesity was included as a covariate (Table S2) and not as an exposure in statistical models.

\section{Conclusions}

Our findings in a broad asthma population initiating SCS, including both acute and maintenance SCS, and followed over a median exposure period of over 7 years indicate that increasing cumulative exposure and increasing mean daily exposure to SCS places patients at a high risk of potentially life-changing SCS-related adverse outcomes. The onset of some outcomes was associated with cumulative SCS exposure of only $0.5-<1 \mathrm{~g}$, equivalent to four lifetime courses of SCS. These findings underscore the importance of improving the awareness of SCS-related adverse effects in patients with asthma and support the need for continuing development and identification of alternative treatments for treating asthma exacerbations and for patients with severe asthma to reduce exposure to SCS. In conclusion, our findings suggest urgent need for reappraisal of when patients 
need specialist care and consideration of effective steroidsparing medications.

\section{Acknowledgments}

This study was funded by AstraZeneca. We thank Aruni Seneviratna and Shreyasee Pradhan for their contributions to the project management for this study and Derek Skinner for his contributions to the data acquisition and handling. Writing and editorial support was provided by Elizabeth V. Hillyer, DVM, supported by the Observational and Pragmatic Research Institute Pte. Ltd (OPRI).

\section{Author contributions}

All authors contributed to the study conception and design, data acquisition, and data analysis and interpretation. All authors contributed to critically revising the manuscript and approved the final version prior to submission. All authors agree to be accountable for all aspects of the work and to ensuring that questions related to the accuracy or integrity of the work are appropriately investigated and resolved.

\section{Disclosures}

DBP has board membership with Aerocrine, Amgen, AstraZeneca, Boehringer Ingelheim, Chiesi, Mylan, Mundipharma, Napp, Novartis, and Teva Pharmaceuticals; consultancy agreements with Almirall, Amgen, AstraZeneca, Boehringer Ingelheim, Chiesi, GlaxoSmithKline, Mylan, Mundipharma, Napp, Novartis, Pfizer, Teva Pharmaceuticals, and Theravance; grants and unrestricted funding for investigator-initiated studies (conducted through OPRI) from Aerocrine, AKL Research and Development Ltd, AstraZeneca, Boehringer Ingelheim, British Lung Foundation, Chiesi, Mylan, Mundipharma, Napp, Novartis, Pfizer, Respiratory Effectiveness Group, Teva Pharmaceuticals, Theravance, UK National Health Service, and Zentiva; payment for lectures/speaking engagements from Almirall, AstraZeneca, Boehringer Ingelheim, Chiesi, Cipla, GlaxoSmithKline, Kyorin, Mylan, Merck, Mundipharma, Novartis, Pfizer, Skyepharma, and Teva Pharmaceuticals; payment for manuscript preparation from Mundipharma and Teva Pharmaceuticals; payment for the development of educational materials from Mundipharma and Novartis; payment for travel/accommodation/meeting expenses from Aerocrine, AstraZeneca, Boehringer Ingelheim, Mundipharma, Napp, Novartis, and Teva Pharmaceuticals; funding for patient enrolment or completion of research from Chiesi, Novartis, Teva Pharmaceuticals, and Zentiva; stock/stock options from AKL Research and Development Ltd, which produces phytopharmaceuticals; owns $74 \%$ of the social enterprise Optimum Patient Care Ltd (Australia and UK) and 74\% of OPRI (Singapore); and is peer reviewer for grant committees of the Efficacy and Mechanism Evaluation program me, and Health Technology Assessment. FT, XX, and TNT are employees and shareholders of AstraZeneca, which supplied the funding for this study. JV and MK are employees of OPRI, and JLZJ at the time of the study was an employee of OPRI, which conducted this study and which has conducted paid research in respiratory disease on behalf of the following organizations in the past 5 years: Aerocrine, AKL Research and Development Ltd, Almirall, AstraZeneca, Boehringer Ingelheim, Chiesi, GlaxoSmithKline, Mylan, Mundipharma, Napp, Novartis, Orion, Takeda, Teva, and Zentiva (a Sanofi company).

\section{References}

1. Fardet L, Petersen I, Nazareth I. Prevalence of long-term oral glucocorticoid prescriptions in the UK over the past 20 years. Rheumatology. 2011;50(11):1982-1990.

2. Bénard-Laribière A, Pariente A, Pambrun E, Bégaud B, Fardet L, Noize P. Prevalence and prescription patterns of oral glucocorticoids in adults: a retrospective cross-sectional and cohort analysis in France. BMJ Open. 2017;7(7):e015905.

3. Sarnes E, Crofford L, Watson M, Dennis G, Kan H, Bass D. Incidence and US costs of corticosteroid-associated adverse events: a systematic literature review. Clin Ther. 2011;33(10):1413-1432.

4. Fardet L, Petersen I, Nazareth I. Common infections in patients prescribed systemic glucocorticoids in primary care: a population-based cohort study. PLoS Med. 2016;13(5):e1002024.

5. Liu D, Ahmet A, Ward L, et al. A practical guide to the monitoring and management of the complications of systemic corticosteroid therapy. Allergy Asthma Clin Immunol. 2013;9(1):30.

6. van Staa TP, Leufkens HG, Abenhaim L, Zhang B, Cooper C. Oral corticosteroids and fracture risk: relationship to daily and cumulative doses. Rheumatology. 2000;39(12):1383-1389.

7. Lefebvre P, Duh MS, Lafeuille MH, et al. Acute and chronic systemic corticosteroid-related complications in patients with severe asthma. J Allergy Clin Immunol. 2015;136(6):1488-1495.

8. Lefebvre P, Duh MS, Lafeuille MH, et al. Burden of systemic glucocorticoid-related complications in severe asthma. Curr Med Res Opin. 2017;33(1):57-65.

9. Daugherty J, Lin X, Baxter R, Suruki R, Bradford E. The impact of longterm systemic glucocorticoid use in severe asthma: A UK retrospective cohort analysis. J Asthma. 2018;55(6):651-658.

10. Waljee AK, Rogers MA, Lin P, et al. Short term use of oral corticosteroids and related harms among adults in the United States: population based cohort study. BMJ. 2017;357:j1415.

11. Fleishaker DL, Mukherjee A, Whaley FS, Daniel S, Zeiher BG. Safety and pharmacodynamic dose response of short-term prednisone in healthy adult subjects: a dose ranging, randomized, placebo-controlled, crossover study. BMC Musculoskelet Disord. 2016;17:293.

12. British guideline on the management of asthma: A national clinical guideline. Revised 2016 (SIGN 153). British Thoracic Society, Scottish Intercollegiate Guidelines Network; September 2016. Available from: http://www.sign.ac.uk/sign-153-british-guideline-on-the-managementof-asthma.html. Accessed March 28, 2018.

13. Sweeney J, Patterson CC, Menzies-Gow A, et al. Comorbidity in severe asthma requiring systemic corticosteroid therapy: cross-sectional data from the Optimum Patient Care Research Database and the British Thoracic Difficult Asthma Registry. Thorax. 2016;71(4):339-346. 
14. Choo XN, Pavord ID. Morbidity associated with oral corticosteroids in patients with severe asthma. Thorax. 2016;71(4):302-304.

15. Sullivan PW, Ghushchyan VH, Globe G, Schatz M. Oral corticosteroid exposure and adverse effects in asthmatic patients. J Allergy Clin Immunol. 2018;141(1):110-116.

16. Optimum Patient Care Research Database (OPCRD). Available from: http://opcrd.co.uk/. Accessed March 28, 2018.

17. Clinical Practice Research Datalink. Available from: http://www.cprd. com/home/. Accessed March 16, 2018.

18. Roche N, Reddel H, Martin R, et al. Quality standards for real-world research. Focus on observational database studies of comparative effectiveness. Ann Am Thorac Soc. 2014;11(Suppl 2):S99-S104.

19. Hospital Episode Statistics (HES). UK National Health Service. Available from: http://content.digital.nhs.uk/hes. Accessed March 16, 2018.

20. Belitser SV, Martens EP, Pestman WR, Groenwold RH, de Boer A, Klungel $\mathrm{OH}$. Measuring balance and model selection in propensity score methods. Pharmacoepidemiol Drug Saf. 2011;20(11):1115-1129.

21. Mickey RM, Greenland S. The impact of confounder selection criteria on effect estimation. Am J Epidemiol. 1989;129(1):125-137.

22. Manson SC, Brown RE, Cerulli A, Vidaurre CF. The cumulative burden of oral corticosteroid side effects and the economic implications of steroid use. Respir Med. 2009;103(7):975-994.
23. Voorham J, Xu X, Price D, et al. Health care resource utilization and costs associated with incremental systemic corticosteroid exposure in asthma. Allergy. Epub 2018 Jul 10.

24. Johnson ES, Bartman BA, Briesacher BA, et al. The incident user design in comparative effectiveness research. Pharmacoepidemiol Drug Saf. 2013;22(1):1-6.

25. Dalal AA, Duh MS, Gozalo L, et al. Dose-response relationship between long-term systemic corticosteroid use and related complications in patients with severe asthma. J Manag Care Spec Pharm. 2016;22(7):833-847.

26. Luskin AT, Antonova EN, Broder MS, Chang EY, Omachi TA, Ledford DK. Health care resource use and costs associated with possible side effects of high oral corticosteroid use in asthma: a claims-based analysis. Clinicoecon Outcomes Res. 2016;8:641-648.

27. Zazzali JL, Broder MS, Omachi TA, Chang E, Sun GH, Raimundo K. Risk of corticosteroid-related adverse events in asthma patients with high oral corticosteroid use. Allergy Asthma Proc. 2015;36(4):268-274.

28. Sullivan PW, Campbell JD, Ghushchyan VH, Globe G. Outcomes before and after treatment escalation to Global Initiative for Asthma steps 4 and 5 in severe asthma. Ann Allergy Asthma Immunol. 2015;114(6):462-469.

29. Walley T, Mantgani A. The UK General Practice Research Database. Lancet. 1997;350(9084):1097-1099.
Journal of Asthma and Allergy

\section{Publish your work in this journal}

The Journal of Asthma and Allergy is an international, peer-reviewed open access journal publishing original research, reports, editorials and commentaries on the following topics: Asthma; Pulmonary physiology; Asthma related clinical health; Clinical immunology and the immunological basis of disease; Pharmacological interventions and
Dovepress

new therapies. This journal is included in PubMed. The manuscript management system is completely online and includes a very quick and fair peer-review system, which is all easy to use. Visit http://www. dovepress.com/testimonials.php to read real quotes from published authors. 